\title{
Fractal theory based dynamic mesh grouping scheme for efficient cognitive pilot channel design
}

\author{
FENG ZhiYong*, WEI ZhiQing, ZHANG QiXun \& ZHANG Ping \\ Wireless Technology Innovation Institute (WTI), Key Laboratory of Universal Wireless Communications, Ministry of Education, \\ Beijing University of Posts and Telecommunications, Beijing 100876, China
}

Received March 6, 2012; accepted June 27, 2012

\begin{abstract}
As one of the key characteristics in cognitive wireless networks (CWNs), network environment awareness techniques have received much attention recently. Instead of traditional spectrum sensing technology that suffers from problems of miss detection, false alarm, hidden node, and inefficiency, cognitive pilot channel (CPC) technology has been proposed as one of the candidate solutions for an efficient and accurate network information delivery scheme to user equipment (UE). The aim of the CPC technology is to provide the necessary information for the reconfiguration of the UE using the public signaling channel. To ensure the efficient information delivery, the whole geographical area covered by the CPC is divided into square meshes of the same size. Moreover, two typical network information transmission modes for CPC deployment are also proposed: broadcast CPC mode and on-demand CPC mode. To further improve the efficiency of network information delivery, an efficient dynamic mesh grouping scheme has been designed which is based on the fractal theory for the broadcast CPC mode, where adaptive rectangular sized meshes are used to approximately cover the whole area. Compared to the traditional fixed size mesh division strategy, results show that the proposed dynamic mesh grouping scheme significantly reduces the number of meshes by grouping similar meshes together, and the average delay of receiving CPC information on the UE side is therefore reduced.
\end{abstract}

cognitive pilot channel, cognitive wireless networks, mesh grouping scheme, region positioning

Citation: Feng Z Y, Wei Z Q, Zhang Q X, et al. Fractal theory based dynamic mesh grouping scheme for efficient cognitive pilot channel design. Chin Sci Bull, 2012, 57: 3684-3690, doi: 10.1007/s11434-012-5369-7

As unveiled by the Federal Communications Commission (FCC) reports in $[1,2]$, precious radio spectrum resources are underutilized, thus greatly hindering the convergence and cooperation of heterogeneous networks. So, the traditional fixed spectrum assignment, which grants exclusive use to licensees or primary users, is unsuitable in the rapidly changing heterogeneous radio environment. New technologies such as Cognitive Radio (CR), which is a contextaware radio with the capability to reconfigure itself to adapt to the changing environment, were first proposed by Mitola in 1998. However, how to gather information from the radio environment is one of the key issues to be solved. As proposed in the cognition cycle [3], the network information gathering process is the first step towards the realization of

*Corresponding author (email: fengzy@bupt.edu.cn)
CR functions.

Based on the concept of $\mathrm{CR}$, the inevitable trend lead to the introduction of Cognitive Wireless Networks (CWNs) as proposed in [4] to extend the physical layer cognition to a network layer cognition with end-to-end goals. In heterogeneous CWNs, with multiple Radio Access Technologies (RATs) coexisting at the same location, collecting information from the environment, which can be classified into multiple domains, such as radio, network, policy, and user domains, is complicated and time consuming. One of the candidate technologies for multiple domain information collection is spectrum sensing, which will face the challenges of time consuming and power consumption when the spectrum bands that need to be sensed are extraordinarily wide. Besides, spectrum sensing can only detect information from the radio spectrum domain, without an efficient multi- 
domain information integration ability. To collect information from the multi-domain environment, the concept of cognitive national database was proposed [5], enabling the user equipment (UE) to receive information about the multidomain environment.

Furthermore, to provide an accurate and efficient transmission channel for UE to retrieve information from the cognitive database, the concept of a Cognitive Pilot Channel (CPC) is proposed, which uses the public signaling channel to transmit multi-domain cognitive environment information, such as the vacant spectrum, available RATs, and spectrum policy in a specific area. In Figure 1, the Heterogeneous Network Information Database (HNID) serves as a cognitive database and the CPC server uses a public transmission channel to deliver information from the database to the UE. As a key enabler for CWNs, the CPC provides a public carrier to collect heterogeneous networks' information and convey it to the UE. Moreover, both the multi-dimension cognitive database and CPC solutions have been accepted and incorporated as candidate technologies for the Cognitive Radio System (CRS) in the land mobile service by the ITU-R WP5A standards organization.

Considering the wide area that needs to be covered by the public signaling channel $\mathrm{CPC}$, the region is divided into square meshes. A mesh is defined as a region in which certain radio electrical commonalities can be identified [6], thereby providing a mechanism for data fusion in the database. In Figure 1, the "mesh" is square, and different UE may be located in the same or different meshes. The cognitive environment information is transmitted to the UE by a CPC transmitter in broadcast or on-demand mode. In broadcast mode, the network information of all meshes is broadcast periodically and continuously, although this may be time consuming with a large number of meshes. The on-demand mode was proposed [6] with both uplink and

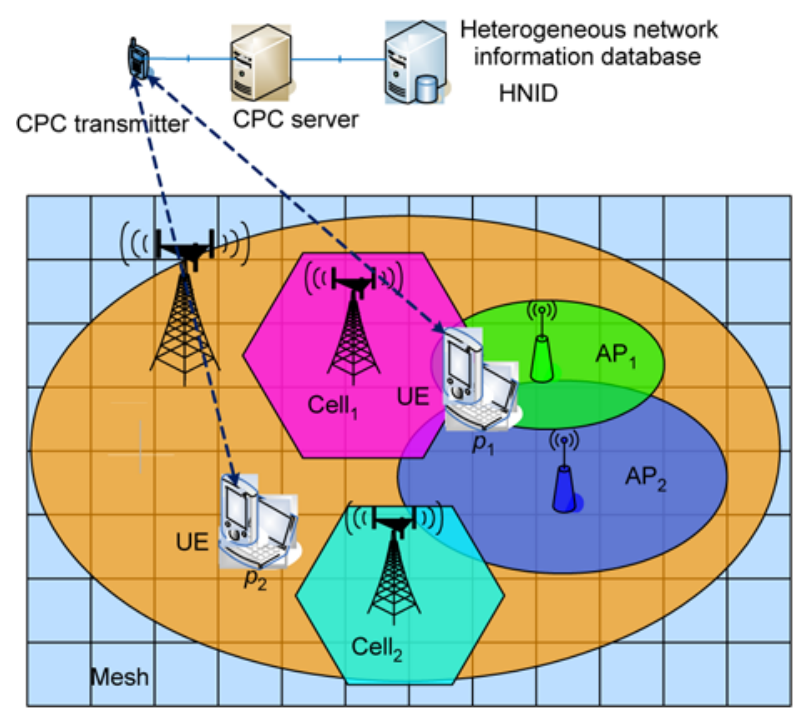

Figure 1 (Color online) Architecture of CPC deployment. downlink components. The average delay for on-demand is smaller than that for broadcast only if the arrival rate of requests is low. When the arrival rate of requests exceeds a certain threshold, the delay for on-demand CPC approaches infinity, corresponding to an unstable queuing system.

Considering the frequency used by CPC in deployment, both out-band and in-band CPC schemes have been proposed. In the out-band CPC scheme, the network information is delivered to the UE within the switch-on period using a common worldwide frequency or a collection of several available frequency bands [7]. On the other hand, the in-band CPC scheme is used to provide more detailed network information in the on-going period [8] by using a specific frequency band of the existing RATs. Both schemes are implemented over a wide area divided into a great number of meshes [7], thus enhancing the accuracy and efficiency of network information delivery.

As cognitive information is categorized by meshes delivered via CPC, mesh management schemes are important for accurate and efficient information delivery. Several studies focusing on mesh management have been reported in the literature. Ref. [6] proposed the concept of mesh division, suggesting meshes with adaptive sizes. Optimal mesh division schemes were designed [9,10], where the scheme in [9] considers the effects of the GPS localization shift error and information loss ratio in a multi-RAT overlapping scenario and improves the accuracy and efficiency of network information delivery. In [10], a novel mesh grouping scheme is presented by grouping the homogeneous meshes logically in the information delivery sequence. However, meshes are not geographically grouped in either [9] or [10]. And more importantly, the frame is lengthened and the transmitterreceiver link needs more bandwidth to implement communication [10]. Therefore, by taking into account the geographical feature of different meshes, a dynamic adaptive size mesh grouping scheme is proposed by grouping the meshes with common information geographically. The proposed novel scheme can significantly reduce the number of meshes and improve the efficiency of information delivery in the broadcast CPC scheme compared with the traditional fixed size mesh scheme.

\section{System model}

As illustrated in Figure 2, different RATs co-exist in a specific area. Usually, one RAT's network may overlap with other RATs. Moreover, the radio environment is complicated, especially in the overlapping area. In Figure 2, five RATs divide the whole area into 11 regions, i.e., $R_{1}, R_{2}, \ldots$, $R_{11}$. Compared with the other regions, each region has different radio parameters due to the specific subset of RATs in its coverage area. For example, $R_{1}$ has only $\mathrm{RAT}_{1}$, whereas $R_{5}$ has $\mathrm{RAT}_{1}, \mathrm{RAT}_{2}$, and $\mathrm{RAT}_{3}$. 


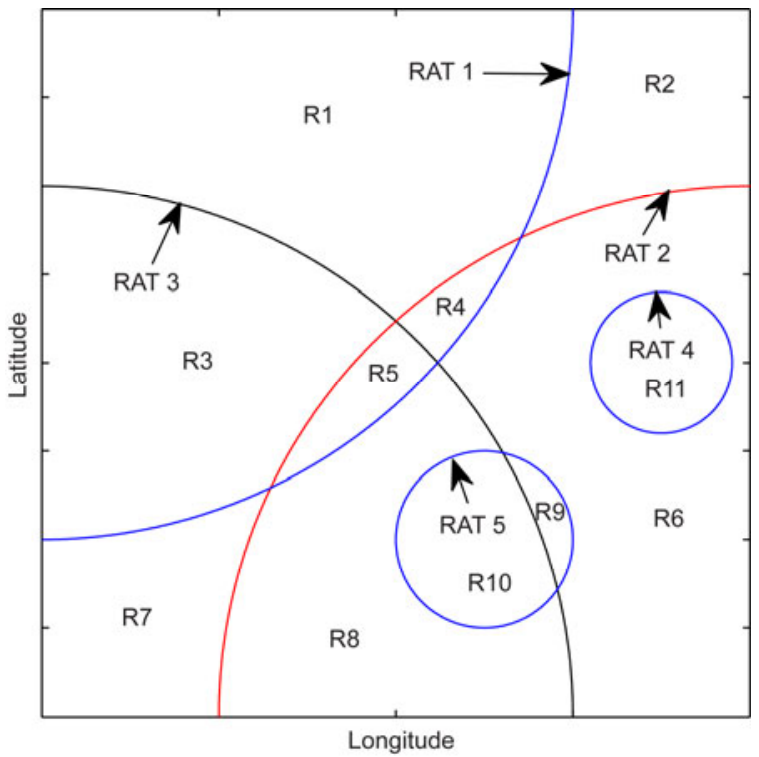

Figure 2 (Color online) Multi-RAT co-existence scenario.

\section{Mesh grouping algorithms}

In this paper, a mesh is defined as a rectangle with the same subset of RATs. The aim of the mesh grouping algorithm is to group a geographic area with homogeneous radio parameters into a mesh. Assume that the mesh's shape is rectangular, and that the proposed mesh grouping algorithm is to group the area with the same subset of RATs into a rectangle.

In contrast to the traditional fixed size mesh division scheme, which divides the whole region into regular small squares as in Figure 1, we divide the whole region into appropriate rectangular meshes using fractal theory, which iteratively divides an unqualified mesh and retains a qualified mesh. Here "qualified mesh" denotes as a mesh with an almost pure radio environment. The algorithm is referred to as the FbMDA (Fractal based Mesh Division Algorithm). After applying the mesh fusion algorithm (referred to as the MFA-aFbMD, Mesh Fusion Algorithm after Fractal based Mesh Division), the homogeneous meshes are further fused. Thus, the whole algorithm is called the FbMD-MGA (Fractal based Mesh Division and Mesh Grouping Algorithm), which is FbMDA followed by MFA-aFbMD. Before explaining the algorithms, the notion of region positioning is first presented below.

\subsection{Notion of region positioning}

Region positioning aims to find a methodology to define a region once a point's geographic information is known, irrespective whether it is known that the point is located in the region.

Obviously, a rectangle can be used in region positioning. Suppose the coordinates of the two vertices on a diagonal are $\left(x_{1}, y_{1}\right),\left(x_{2}, y_{2}\right)$ with $x_{1}<x_{2}, y_{1}<y_{2}$. Let the point's coordi- nates be $(x, y)$, then the point is located in the rectangle, iff

$$
x_{1}<x<x_{2} \text { and } y_{1}<y<y_{2} .
$$

Thus, a rectangle can implement region positioning for its position localization. In this paper, a mesh is denoted as $\left[x_{1}, y_{1} ; x_{2}, y_{2}\right]$, as illustrated in Figure 3. Generally speaking, the convex hull of a set of points in a region can be used to locate this region. But the geographic information of a convex hull may be complex and is not practical in processing. Some simpler graphs, such as rectangle and triangle, are preferred in region positioning.

\subsection{FbMD-MGA}

(i) Discrete processing. Divide the whole area equally into $N \times N$ small rectangles, where $N=2^{k}$, with $k$ a positive integer. Now obtain the coordinates of the center of each small rectangle. Assume that the radio environment parameters of any point in the small rectangle are the same as that of the center. Thus, the center is representative of the small rectangle within which it is located. In other words, the small rectangle is the minimum spatial resolution during processing, henceforth called a "pixel". Define the binary representation of $\mathrm{RAT}_{i}$ in $(x, y)$ as follows.

$$
M\left(\mathrm{RAT}_{i}, x, y\right)= \begin{cases}1 & \text { if } \mathrm{RAT}_{i} \text { is detected in }(x, y), \\ 0 & \text { if } \mathrm{RAT}_{i} \text { is not detected in }(x, y) .\end{cases}
$$

Using the above representation, it is possible to characterize geographical coordinates by a value corresponding to the sum of the binary representations of all the RATs at this point [6]:

$$
I(x, y)=\sum_{i=1}^{T} M\left(\operatorname{RAT}_{i}, x, y\right) \times 2^{i-1},
$$

where $T$ is the number of RATs. The $I(x, y)$ of each pixel's center is defined as the feature of the pixel, and is stored in an $N \times N$ matrix, denoted as Gra, which can characterize the

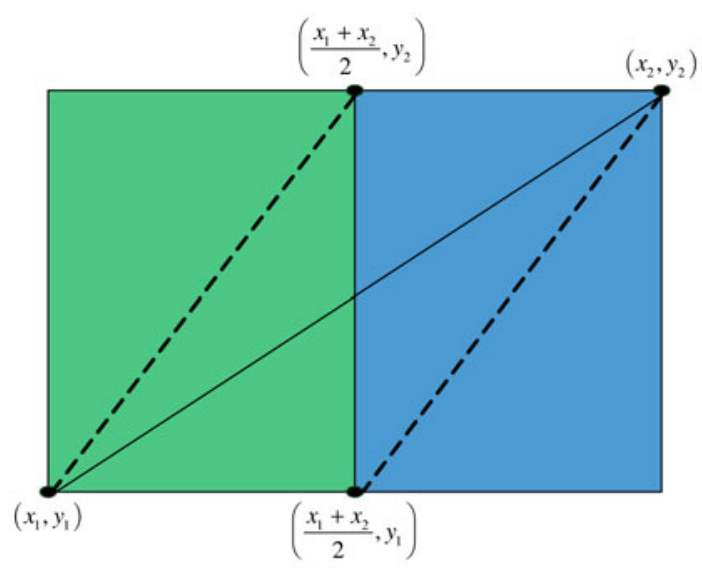

Figure 3 (Color online) Mesh division. 
whole geographic area. Note that $I(x, y)$ is an integer range between 0 and $2^{T}$, where $T$ is the number of RATs.

(ii) Parameters of FbMDA. Three basic parameters are addressed, namely, error limit, minimum edge of mesh, and stack of unqualified meshes, which are defined as follows.

Error limit: Denoted as $\sigma_{\mathrm{min}}$. Let the Radio Parameters Error (denoted as RPE) of a rectangle be defined as in eq. (4).

$$
\mathrm{RPE}=1-\max _{J}\left\{\frac{N_{J}}{N_{P}}\right\},
$$

where $N_{J}$ is the number of pixels whose features are $J$ according to eq. (3), and $N_{P}$ is the total number of pixels in the rectangle. If a rectangle's RPE is smaller than $\sigma_{\max }$, it is a qualified mesh, which means that the radio environment of this rectangle is almost pure.

Minimum edge of mesh: Denoted as $e_{\min }$, this is the length of the edge of the minimum mesh. That is, the minimum mesh divided. If a rectangle's edge is shorter than $e_{\min }$, it is also qualified, which means the mesh cannot be divided again.

Stack of unqualified meshes: This stores unqualified meshes. At each iteration, the algorithm divides an unqualified mesh taken from the stack, and two new meshes emerge. The qualified meshes are stored in an array, while the unqualified meshes are stored in the stack for the next iteration.

(iii) FbMDA description. The FbMDA is described in Algorithm 1, where the mesh is denoted by the coordinates of the vertices on the diagonal, as well as the whole area, which is the first unqualified mesh. The division operation is illustrated in Figure 3, where mesh $\left[x_{1}, y_{1} ; x_{2}, y_{2}\right]$ is divided into two smaller meshes as in eq. (5).

$$
\left[x_{1}, y_{1} ; \frac{x_{1}+x_{2}}{2}, y_{2}\right],\left[\frac{x_{1}+x_{2}}{2}, y_{1} ; x_{2}, y_{2}\right] \text {. }
$$

\subsection{Analysis of mesh division algorithm}

The algorithm can be described as a form of binary tree. As illustrated in Figure 4, mesh 1 comprises the whole region, which is then divided into meshes 2 and 3 . Mesh 3 is a qualified mesh, whereas mesh 2 is unqualified. Since we iteratively divide the unqualified mesh and retain the qualified mesh, mesh 3 is retained while mesh 2 is divided into meshes 4 and 5. This process is repeated until the whole region is divided into qualified meshes, as illustrated in Figure 4(b).

According to the FbMDA, the binary tree has the following properties.

Theorem 1. No mesh has only one child.

Proof: According to the FbMDA, if a mesh is qualified, it has no child. If a mesh is unqualified, it has two children, as it is divided into two smaller meshes.

Theorem 2. The area of the mesh in the $i$ th layer of the

\section{Algorithm 1 FbMDA}

Initialize $\sigma_{\min }, e_{\min } ;$ stack $\leftarrow$ wholearea, array $\leftarrow \phi$
while stack is not empty do
Pop mesh $m$ from stack
Divide $m$, take two smaller meshes $m_{1}, m_{2}$.
for $j=1$ to 2 do
if $m_{j}$ is qualified then
Append $m_{j}$ to array
else
Push $m_{j}$ in stack
end if
end for
end while

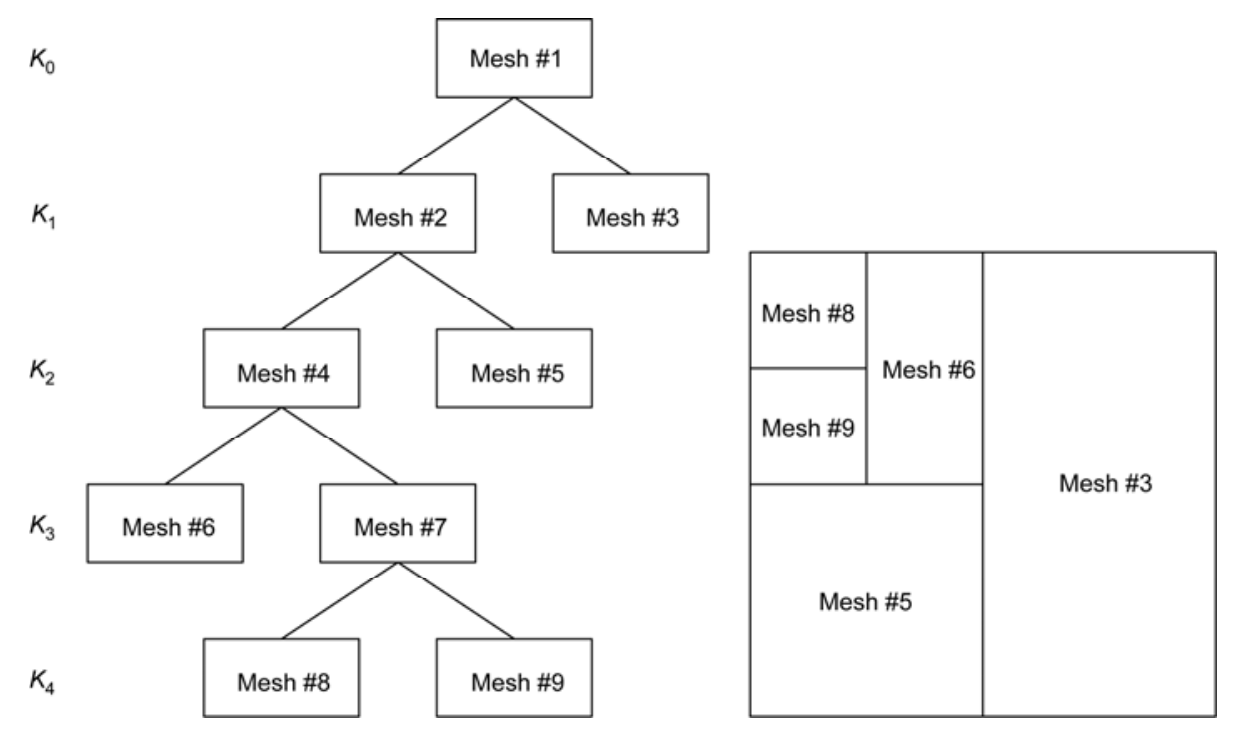

Figure 4 Fractal based mesh division. 
binary tree is $S_{i}=S / 2^{i}$, where $S$ is the area of the whole region.

Proof: The mesh in the $i$ th layer is divided by $i$ times, and thus the area thereof is $S / 2^{i}$.

Theorem 3. Denote the number of qualified meshes in the $i$ th layer as $K_{i}$, then $\sum_{i=1}^{N} K_{i} 2^{-i}=1$, where $N$ is the number of iterations (layers).

Proof: The sum of the areas of all the qualified meshes is $S$, and so we have $\sum_{i=1}^{N} K_{i} S_{i}=S$. According to Theorem 2, $S_{i}=S / 2^{i}$, and therefore

$$
\sum_{i=1}^{N} K_{i} \frac{S}{2^{i}}=S \Rightarrow \sum_{i=1}^{N} \frac{K_{i}}{2^{i}}=1 .
$$

Thus, we have Theorem 3.

Theorem 4. A qualified mesh is the leaf of the binary tree.

Proof: A mesh is qualified iff the mesh cannot be divided again (i.e., has no child), and thus it is definitely a leaf of the binary tree.

Theorem 5. If the binary tree has Nlayers (we do not count the 0th layer), then the number of qualified meshes varies from $N+1$ to $2^{N}$.

Proof: This theorem is simple and crucial. According to Theorem 3, we have

$$
1=\sum_{i=1}^{N} \frac{K_{i}}{2^{i}} \geqslant \sum_{i=1}^{N} \frac{K_{i}}{2^{N}}=\frac{1}{2^{N}} \sum_{i=1}^{N} K_{i} .
$$

Thus, we have the upper bound of the number of qualified meshes

$$
\sum_{i=1}^{N} K_{i} \leqslant 2^{N}
$$

Let the number of meshes with two children be $n_{2}$, and the number of meshes with no children be $n_{0}$. By Theorem 1 , none of the meshes has only one child. According to the property of binary trees, we have $n_{0}=n_{2}+1$. And note that once a mesh has been divided, the value of $n_{2}$ increases by 1 . Thus, to stop the increase in $n_{2}$, we must reduce the number of division operations. Dividing one mesh in one layer is the most economic operation, which outputs a mesh with two children. Finally, we have $n_{2}=N$ and $n_{0}=n_{2}+1=N+1$.

\subsection{Analysis of radio parameter errors}

RPE defined in eq.(4) is the radio parameter error of one mesh. We denote the RPE of mesh $i$ as $p_{e, i}$, while the RPE of the whole region is defined as

$$
p_{e}=\sum_{i=1}^{M} \frac{s_{i}}{S} p_{e, i}=\sum_{i=1}^{M} \frac{s_{i}}{S}\left(1-\max _{j} p_{i j}\right),
$$

where $s_{i}$ is the area of mesh $i$, and $S$ is the area of the whole region. We linearly combine each $p_{e, i}$ to obtain $p_{e}$ with $\frac{s_{i}}{S}$ the weight. We have $p_{e, i}=1-\max _{j} p_{i j}$, where $p_{i j}$ is the proportion of the area with feature $j$ in mesh $i$. This formula is essentially the same as eq. (4), as we define the feature of mesh $i$ as

$$
J=\arg \max _{j} p_{i j}
$$

and eq. (6) is reasonable. We have $\max _{j} p_{i j} \geqslant \frac{1}{2^{T}}$, where $T$ is the number of RATs and the upper bound of $p_{e, i}$ is $p_{e, i} \leqslant 1-\frac{1}{2^{T}}$.

If the number of meshes $M$ is sufficiently large, the meshes with impure radio parameters are distributed along the boundary of the RATs. Denoting the length of all the RATs' boundaries as $\xi$, the length of a mesh's edge as $\varepsilon$, and the length of the whole region's edge as $L$, we have $M=\left(\frac{L}{\varepsilon}\right)^{2}$. Regular mesh division is assumed and the upper bound of the number of meshes with impure radio parameters is

$$
K \leqslant \frac{2 \xi \sqrt{2} \varepsilon}{\varepsilon^{2}}=\frac{2 \sqrt{2} \xi}{\varepsilon} .
$$

The upper bound in eq. (8) is derived by solving a packing problem. As illustrated in Figure 5, the solid line is the boundary of all the RATs. Each point on the solid line is translated $\sqrt{2} \varepsilon$ in the two normal directions, thus revealing the two dotted lines in Figure 5, the area between which is $2 \xi \sqrt{2} \varepsilon$. As all the meshes with impure radio parameters are distributed between the two dotted lines, the upper bound of $K$ is calculated as $2 \xi \sqrt{2} \varepsilon$ divided by the area of a mesh.

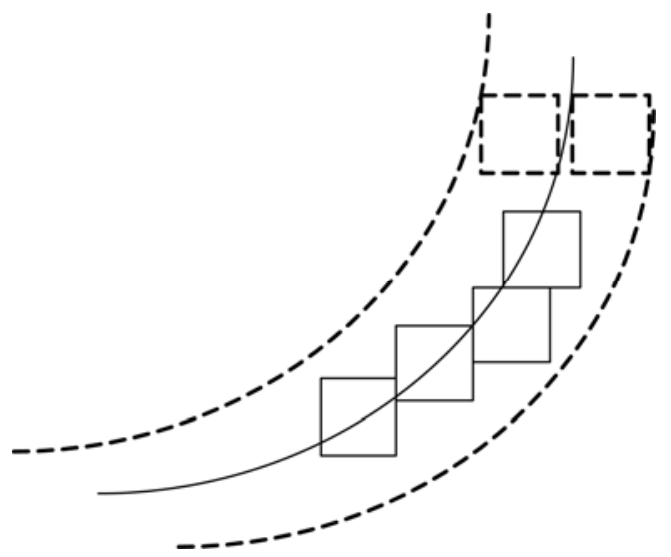

Figure 5 A packing problem to derive the upper bound of $K$. 
As regular mesh division is assumed, we have $\frac{s_{i}}{S}=\frac{1}{M}$, and the upper bound of $p_{e}$ is derived by eqs. (6) and (8).

$$
p_{e} \leqslant \frac{1}{M} K\left(1-\frac{1}{N}\right)=\frac{1}{\sqrt{M}} \frac{2 \sqrt{2} \xi L}{S}\left(1-\frac{1}{N}\right) .
$$

It is obvious that $\lim _{M \rightarrow \infty} p_{e}=0$, and we have Theorem 6 .

Theorem 6. The radio parameter error of the whole region is reduced to 0 with rate $\mathrm{O}\left(\frac{1}{\sqrt{M}}\right)$ as $M$ increases.

Thus, an increase in $M$ can reduce the radio parameter error by rate $\mathrm{O}\left(\frac{1}{\sqrt{M}}\right)$, but this will increase the delay of CPC information transmission. So an interesting tradeoff emerges.

\subsection{The fusion operation}

Homogeneous meshes after applying FbMDA do exist. So FbMDA followed by the MFA (Mesh Fusion Algorithm) yields better performance. If the MFA is not applied, FbMDA alone is also applicable.

If two meshes are fusible, they are not only geographically adjacent, but also have the same radio environment. A mesh's radio environment is quantified as the feature of the majority pixels according to eq. (7), and is also denoted as the feature of the mesh. Fusing mesh $_{i}$ and mesh $_{j}$ into one is equivalent to an operation that fuses two rectangles into one. Meshes $\left[x_{1}, y_{1} ; x_{2}, y_{2}\right]$ and $\left[x_{3}, y_{3} ; x_{4}, y_{4}\right]$ can be fused into mesh $\left[\min x_{i}, \min y_{i}\right.$; $\left.\max x_{i}, \max y_{i}\right]$. Moreover, if $\operatorname{mesh}_{i}$ and mesh $_{j}$ are fusible, the relation is denoted as " mesh $_{i} \leftrightarrow$ mesh $_{j}$ ". Algorithm MFA-aFbMD finds the fusible meshes in each iteration and fuses them into one mesh, which is described in Algorithm 2.

\section{Results and analysis}

In discrete processing, let $N=1024, \sigma_{\max }=10 \%$, and $e_{\min }=$ $32 e_{\text {pixel }}$, where $e_{\text {pixel }}$ is the length of a pixel's edge. Let the time to transmit the information of a mesh be $20 \mathrm{~ms}$, and users are distributed uniformly in a rectangle area with density ranging from 10 to 2000 users per $\mathrm{km}^{2}$. Assume that users are always synchronized with the CPC channel and that a user randomly requests the CPC information with equal probability [6]. With these parameters, we simulated the mesh grouping algorithms and the broadcasting procedure.

As shown by the mesh division and fusion results in Figure 6 , the total number of meshes is reduced after the fusion

\section{Algorithm 2 MFA-aFbMD}

Get mesh number $L$; label $\leftarrow$ true
while label do
label $\leftarrow$ false, $i \leftarrow 1$, take mesh $_{i}$
while $i \leqslant L$ do
$j \leftarrow 1$
while $j \leqslant L$ do
if mesh $_{j} \leftrightarrow$ mesh $_{i}$ then
$\quad$ fuse mesh and mesh $_{i}$
$\quad$ Update array mesh, label $\leftarrow$ true
end if
$j=j+1$
end while
$i=i+1$

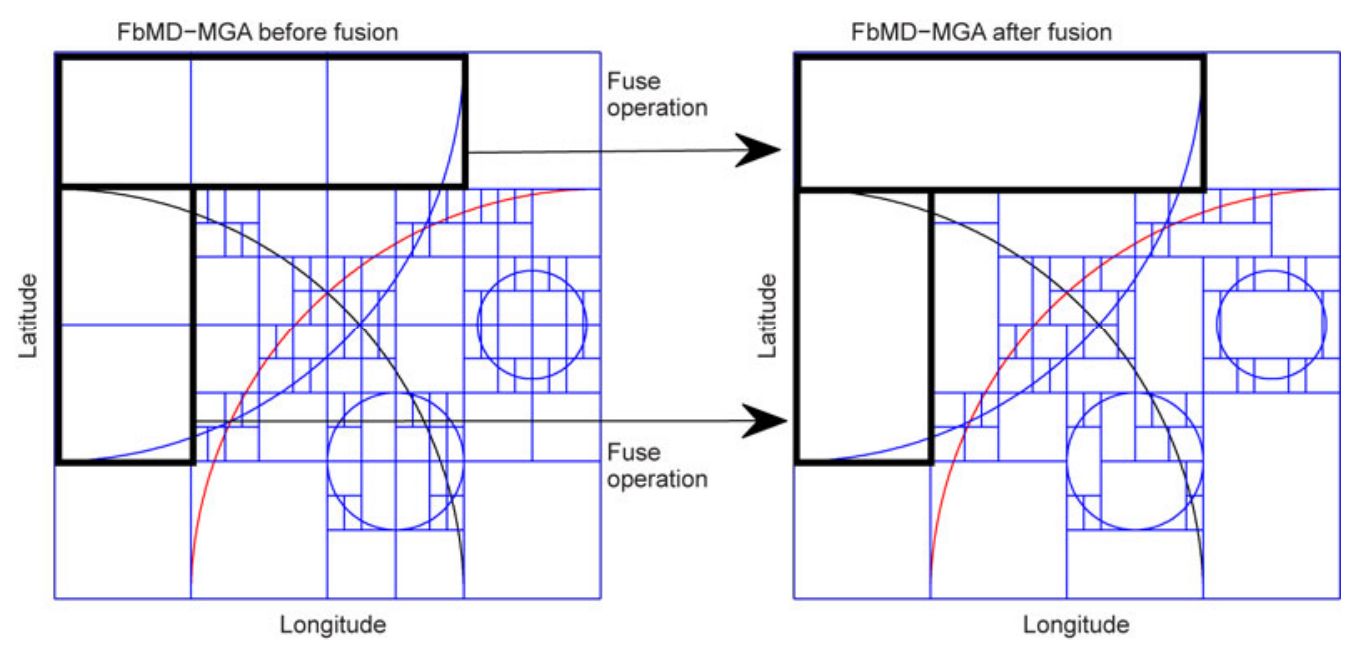

Figure 6 (Color online) Mesh grouping results. 
operation. To ensure the RPE requirement, a CPC without mesh grouping has $(N / 32)^{2}=1024$ meshes, while the number of meshes for FbMD-MGA before fusion is 113 (Figure 6(a)), and that for FbMD-MGA after fusion is 70 (Figure 6(b)). The fusion operation can fuse the meshes with the same feature into one, which can reduce the number of meshes. But the number of fusible meshes after FbMDA is small, so the reduction of the number of meshes is not significant. Actually, the FbMDA alone without fusion operation is also applicable.

When the total number of meshes is reduced, the delay is also reduced, as illustrated in Figure 7. Actually, the average delay for receiving CPC information on the user side is $D_{B}=T_{m, B}\left(\frac{N_{m}}{2}+1\right)$ in [6], where $T_{m, B}$ is the time to transmit the information of a mesh and $N_{m}$ is the number of meshes. It's obvious that the reduction of $N_{m}$ can correspondingly reduce the delay of CPC information transmission.

\section{Conclusion}

To improve the accuracy and efficiency of cognitive information delivery in CWNs, the concept of CPC was proposed.

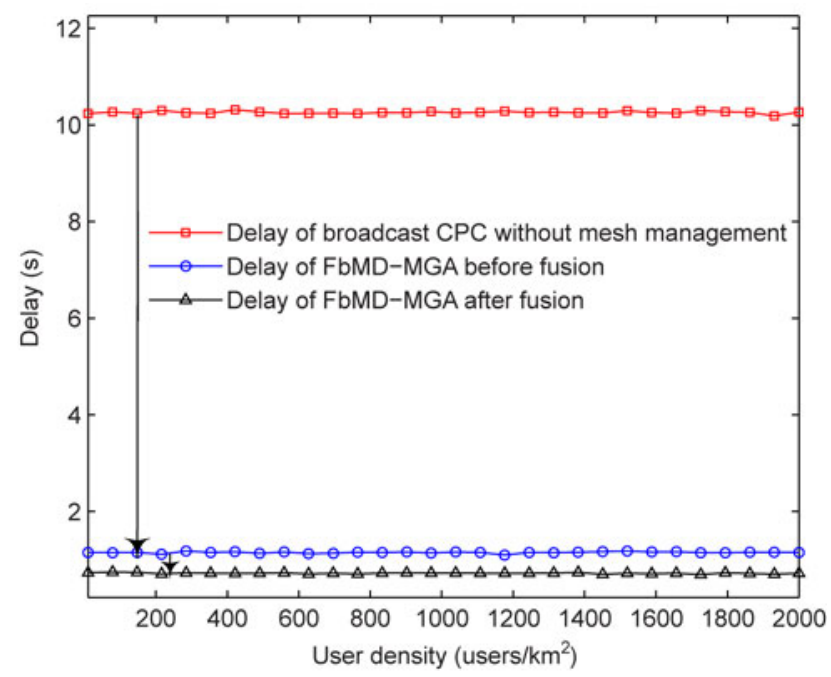

Figure 7 (Color online) Delay of each scheme.
In this paper we introduced a dynamic mesh grouping scheme with adaptive mesh size for CPC, which can significantly reduce the number of meshes and transmission delay. As an efficient scheme for information awareness in CWNs, the proposed dynamic mesh grouping scheme provides a novel data fusion mechanism, which can be used in a cognitive database. Besides, we analyzed the mathematical properties of mesh division algorithm and the radio parameter errors. And some theorems are proposed to establish the mathematical foundation of mesh grouping algorithms.

This work was supported by the National Basic Research Program of China (2009CB320400), the National Natural Science Foundation of China (60832009, 61121001), the Program for New Century Excellent Talents in University (NCET-01-0259), the National Science and Technology Major Project of China (2012ZX03003006).

1 FCC, Spectrum Policy Task Force, FCC Document ET Docket No. 02-135, 2002

2 FCC, Facilitating opportunities for flexible, efficient and reliable spectrum use employing cognitive radio technologies: Notice of proposed rulemaking and order, FCC Document ET Docket No. 03-108, 2003

3 Mitola J, Maquire J, Gerald Q. Cognitive radio: Making software radios more personal. IEEE Pers Commun, 1999, 6: 13-18

4 Feng Z, Zhang Q, Tian F, et al. Novel research on cognitive pilot channel in cognitive wireless network. Wireless Pers Commun, 2012, 62: 455-478

5 FCC and states jointly develop a Nationwide Database of broadband deployment activities, http:transition.fcc.gov/Bureaus/Common_Carrier/ News_Releases/2000/nrcc0030.html

6 Perez-Romero J, Salient O, Agusti R, et al. A novel on-demand cognitive pilot channel enabling dynamic spectrum allocation. In: Proceedings of the IEEE International Symposium on Dyspan, $2007 \mathrm{Apr}$ 17-20, Dublin, Ireland. Washington DC: IEEE, 2007

7 Filo M, Hossain A, Biswas A, et al. Cognitive pilot channel: Enabler for radio systems coexistence. In: Proceedings of the Second International Workshop on Cognitive Radio and Advanced Spectrum Management, 2009 May 18-20, Aalborg, Denmark. Washington DC: IEEE, 2009

8 Sallent O, Pérez-Romero J, Agustí R, et al. Cognitive pilot channel enabling spectrum awareness. In: Proceedings of IEEE International Conference on Communications Workshops, 2009 Jun 14-18, Dresden, Germany. Washington DC: IEEE, 2009

9 Zhang Q, Feng Z, Zhang G, et al. Efficient mesh division and differential information coding schemes in broadcast cognitive pilot channel. Wireless Pers Commun, 2012, 63: 363-392

10 Zhang Q, Feng Z, Zhang G, et al. A novel homogeneous mesh grouping scheme for broadcast cognitive pilot channel in cognitive wireless networks. In: Proceedings of IEEE International Conference on Communications, 2010 May 23-27, Cape Town, South Africa. Washington DC: IEEE, 2010

Open Access This article is distributed under the terms of the Creative Commons Attribution License which permits any use, distribution, and reproduction in any medium, provided the original author(s) and source are credited. 